\title{
Fútbol y racismo: estudio exploratorio en la hinchada del América de Cali
}

\author{
Juan Sebatian Castillo \\ Alejandra Zuluaga \\ (juansebastiancastillocastro@gmail.com) (aleja2_06@hotmail.com) \\ Juan José Serrano Rojas \\ (juan.serrano@correo.icesi.edu.co)
}

Artículo corto de investigación recibido el 26/01/2016 y aprobado el 19/05/2016.

Cómo citar este artículo:

CASTILLO, Juan Sebastián; ZULUAGA, Alejandra; y SERRANO ROJAS, Juan José (2016). "Fútbol y racismo: estudio exploratorio en la hinchada del América de Cali”. En: Trans-pasando Fronteras, Núm. 10, pp. 57-66. Cali, Colombia: Centro de Estudios Interdisciplinarios, Jurídicos, Sociales y Humanistas (CIES), Facultad de Derecho y Ciencias Sociales, Universidad Icesi.

\section{Resumen}

Este artículo rastrea la idea del estadio de fútbol como un espacio en el cual las personas impulsivamente se refieren al "Otro", naturalizando el uso de la violencia verbal hacia los adversarios, e incluso contra los mismos jugadores de la hinchada local. Para ello, se propone describir cómo se enmarcan las prácticas y discursos racistas en este escenario deportivo a partir de la observación participativa de seis partidos del América de Cali, tres de ellos jugando de visitante y tres de local.

\section{Palabras clave:}

Fútbol, Racismo, América de Cali, Hinchada, la otredad 


\section{Introducción}

El fútbol es el deporte que más toca las fibras sentimentales en el mundo. Este reúne a lo largo del año y del mundo la mayor cantidad de espectadores para ir a ver jugar al equipo de su preferencia. Tanto para quienes aman el fútbol, y quienes no, reconocen que el fútbol desborda pasiones, mueve masas y despierta más sentimientos que otros escenarios de la vida pública. En este escenario se enmarca la ciudad de Cali, una ciudad de más de 2 millones de habitantes, y con tres equipos profesionales: América de Cali, el Deportivo Cali y Atlético Fútbol Club. ${ }^{1}$

Ahora bien, las personas describen el estadio como un lugar donde todos son iguales, afuera quedan las diferencias políticas, sociales y económicas. Parcialmente esto parece real, se puede ejemplificar cuando juega la selección que se "olvidan los problemas nacionales y las diferencias políticas" que tienen al país tan dividido. Cada gol es la oportunidad perfecta para abrazar a la persona que se tiene al lado, sea quien sea.

Sin embargo, hay prácticas muy cotidianas que se han ido construyendo y fortaleciendo en el estadio como los discursos racistas. El fútbol se presenta como el escenario perfecto en el cual se está creando una realidad totalmente contradictoria "puede convertirse en fuente de integración social en la lucha contra el racismo (...) o por el contrario, puede ser un medio de la discriminación racial de acuerdo con la filosofía del sistema competitivo que parece en algunos casos incompatible con la inclusión y participación" (González y Jiménez, 2006:69).

En el presente texto se plantea la tesis que el estadio se consolida como un espacio en el cual las personas impulsivamente se refieren al Otro. En este espacio, se crea una naturalización del uso de la violencia verbal hacia los adversarios, e incluso contra los mismos jugadores de la hinchada local. Para abordar la anterior tesis, se propone en el presente escrito describir cómo se enmarcan prácticas y discursos racistas en este escenario deportivo. Para lograr la anterior, se describe el marco teórico; luego, se presenta la metodología y la delimitación y, por último, los hallazgos.

1 El Atlético Fútbol Club, otrora Dépor Aguablanca Fútbol Club, intentó modificar su razón social a Dépor Fútbol Club (Dépór FC), pero por pérdida de su reconocimiento deportivo por parte de Coldeportes, a raíz de irregularidades durante el proceso compra de la ficha del Club Real Sincelejo en el 2004 (Tiempo, 2015), tuvo que disitir de ello, para luego cambiar a la que lleva actualmente (El País, 2016). 


\section{Marco teórico}

El tema de estudio nace por dos motivos principales: el primero, por la intención de analizar un espacio que es comúnmente aceptado por una gran mayoría de la población colombiana y del mundo: el fútbol. El segundo respondiendo al llamado que hace Maguirre (2004) citado por González (2006) quien está describiendo que la aproximación científico social a la cuestión del racismo y el deporte es algo escasa y superficial, por ende, se necesita profundizar mucho más tanto a nivel teórico como empírico; ahora bien, se debe aclarar que este llamado ha sido abordado por diferentes autores ${ }^{2}$ en Europa. Sin embargo, en Colombia es un tema poco abordado, por ende, al asistir frecuentemente era la oportunidad adecuada para aplicar los conocimientos adquiridos en el curso Debates contemporáneos I.

Para abordar el análisis de carácter descriptivo, partimos de una de las premisas importantes del texto de Mills (1997) y es que durante muchos años discursos como los del progreso y de la modernización han sido utilizados para justificar la dominación de los blancos. Esta premisa es importante, porque, en cierta medida, en el fútbol está sucediendo algo similar, González (2006) identifica puntos importantes para entender cómo se relaciona lo que está diciendo Mills con el fútbol, y es que las políticas deportivas y culturales están pensadas desde la óptica que es un escenario en el cual las manifestaciones promueven la inclusión social, y se da por hecho que es útil contra la exclusión social, el racismo, la pobreza o la segregación. Él lo identifica como una visión idealizada o políticamente correcta que han creado, pero realmente lo mira como una realidad dialéctica, y que puede ser ese escenario de inclusión y celebración pacífica de la diferencia, o por el contrario, puede ser un medio de discriminación racial en el marco de un deporte tan competitivo. Es decir, el discurso de inclusión que se ha creado alrededor del deporte, particularmente el fútbol, está convirtiéndose en el escenario para que puedan decir es que hay igualdad, se respeta la diferencia porque para eso está pensado el espacio; pero a la vez, se sigue reproduciendo un discurso racista que lo camuflan bajo la anterior premisa.

2 Unesco (2015); González (2006); Lapchick (2005); Raxen (2005) Sage, G.H. (2001) entre otros. Cabe destacar que muchos de los autores sus análisis apuntan a entender cómo se están configurando los racismos y sus formas en el deporte; algunos sí lo han ampliado al campo del fútbol. Pero estos autores dan luz de cómo abordar el presente estudio 
Complementando lo anterior, Rodolfo Muñoz (2009) resalta que por medio del futbol se han estimulado la formación de identidades colectivas, ya que se construyen gracias al vínculo implícito que hay entre los equipos y comunidades. Las cuales se consolidan desde las significaciones y representaciones que tienen las prácticas de los jugadores y de los hinchas - de un mismo equipo- que se integran en los escenarios deportivos. Sin embargo, las dinámicas del futbol son condicionadas por la gran cantidad de dinero y de intereses que confluyen en cada encuentro; hay 3 aportes significativos, el pago realizado por parte de los asistentes a los partidos, el dinero recibido por la contratación de jugadores, y los pagos efectuados por la publicidad a través de la televisión (Muñoz 2009).

Razón por la cual, se puede considerar que en el futbol hay una lógica comercial agresiva, que a la vez está acompañada por las dinámicas propias de la competitividad, incluyendo relaciones de poder, que se evidencian de manera más simple en un escenario como el estadio. Puesto que, aquel que resulta ganador obtiene más poder, reafirmando el tipo de pertenencia e identidad, con el color de la camiseta, de una región y de una clase social. De la misma manera, el ganador obtiene una cantidad de recursos significativa para el club (Muñoz 2009). Por ende, es claro que la ideología está presente en el futbol y en el estadio, en donde las dinámicas mencionadas generan choques verbales o físicos, que pueden incluir manifestaciones racistas para deslegitimar al rival, las cuales también pueden ser producto, según Muñoz (2009) del vacío y/o frustración que se produce en los actores por falta del estímulo (ganar) que confirma supremacías y poderes.

Por otro lado, organizaciones como la UNESCO (2016) ha descrito tres formas de racismo y discriminación: el racismo impulsivo que son expresiones verbales debido a los sentimientos generados en el aficionado; el segundo, racismo instrumental que es el uso selectivo del racismo y del discurso discriminatorio que no necesita respaldo de una convicción ideológica o creencia; y por último, el racismo institucional entendido como prácticas que no son explicitas pero sí son institucionales.

A lo anterior, se le debe añadir el análisis que también hace la UNESCO (2016) para describir un contexto internacional del racismo y el fútbol, por decirlo de alguna manera. Cabe decir que Colombia no es un caso aislado donde se presentan actitudes racistas y discriminatorias. Europa, cuna de la fiebre por la pasión futbolista de las grandes ligas, en los años 70s empezaron a presentar actitudes racistas y xenofóbicas en la sociedad, pero 
con la ola de llegada de inmigrantes en los 90, se conformó una composición multicultural en los campos de futbol de los diferentes equipos del viejo continente, suscitando un aumento en los problemas de racismo y discriminación. Por esto, surgen en Europa muchas organizaciones, campañas e iniciativas en contra del racismo, la discriminación y la xenofobia en el futbol y en cambio apostaron por una inclusión e igualdad social mediante este deporte (UNESCO, 2016; 21-22). Desafortunadamente, hoy día estos problemas no se han logrado mitigar del todo en un deporte tan común como lo es el futbol.

Además de lo que señala Raxen (2005) que el fútbol se convierte, en ocasiones, en el espacio en que diferentes manifestaciones confluyen. Pese a que se han llevado a cabo diferentes pactos para promover un trato más amable y ameno, y permitir que el futbol se convierta en un espacio de disfrute del deporte, siguen existiendo manifestaciones de violencia, intolerancia, racismo y exclusión de algunos grupos.

\section{Metodología y delimitación}

Ahora bien, para el presente trabajo se apeló a una observación participativa en dos momentos relevantes: el primero, cuando América jugaba de local asistíamos al estadio; y cuando jugaba de visitante cada uno veía el partido en lugares diferentes. Ahora bien, cabe destacar que los comentarios y análisis que se hacen en el presente documento responden a seis partidos observados por los tres investigadores, de ellos tres de visitante y tres de local. ${ }^{3}$ También se tiene en cuenta nuestra experiencia ${ }^{4}$ tras ir constantemente al estadio y hacer un ejercicio de memoria con la óptica del presente trabajo. Es importante destacar que en este trabajo no sé está apuntando a afirmar que la hinchada del América de Cali es racista o no lo es, ni tampoco que estamos abordando todas las prácticas y discursos racistas que se dan en el Pascual Guerrero. Nuestro trabajo es exploratorio, para observar cómo está sucediendo esto. Lo anterior, por cuestiones de tiempo y costos, será realizado a partir de la óptica de una sola tribuna -oriental segundo piso y primer piso-, además de tres espacios diferentes cuando

3 De visitante: días 25 de octubre en el Estadio de Armenia; 13 de noviembre en el estadio de Cartagena;20 de noviembre en el estadio de Popayán; de local: 01, 06 y 27 de noviembre en el Pascual Guerrero. Además de los anteriores - que son los de cuadrangulares de ascenso-, también se asistió a la final del torneo águila, el día 10 de diciembre de 2016.

4 Dos de los tres investigadores van constantemente al estadio a ver al América 
juega de visitante ya que solo somos tres quienes participamos en la investigación. Sin embargo, estamos intentando, de manera muy superficial, aportar al análisis empírico desde lo teórico de cómo se está manifestando en el fútbol colombiano prácticas y discursos racistas, desde la hinchada americana, y atendiendo al llamado de varios escritos de la necesidad de escribir sobre este tema.

\section{Hallazgos}

Observamos que la hinchada en el estadio juega un papel fundamental respecto a la motivación que puede impartir al equipo, o la desconcentración que puede fomentar al equipo contrario. Los mismos clubes y asociaciones de fútbol lo reconocen, de tal manera que en muchas competiciones oficiales, los goles en condición de visitante tienen más valor.

¿Cuál es la relación de las microinteracciones caracterizadas anteriormente y las manifestaciones racistas? durante la observación participante se escuchaba constantemente gritos y reclamos que salían de manera fluida hacia jugadores afrocolombianos que por lo general venían acompañados de palabras denigrantes. Ante lo cual sorprendía, la ausente reacción de los demás espectadores frente a estos comentarios.

Ahora bien, ¿por qué se convierte el estadio en un lugar donde no está mal visto ser racista? ${ }^{5}$ Según Elías y Dunning citados por UNESCO (2015) el fútbol es comparable con la guerra, en cuanto a aquellos instintos agresivos y emocionales que suelen desatarse en esta. No obstante, es claro que en el fútbol solo se busca una dominación temporal del enemigo, y existe una necesidad de menosprecio del oponente que se desarrolla a partir de la violencia verbal, con ánimo de desestabilizar al adversario. Razón por la cual, las expresiones racistas agresivas y denigrantes contra los oponentes salen a flor de piel, sin generar reacciones en los demás asistentes. Asimismo, ver el fútbol como un deporte donde se desatan los impulsos libidinosos, afectivos y emocionales, en cierta medida justifica porque es usual que se presenten expresiones racistas, sin embargo esto no quiere decir, que al nosotros identificar que esta noción se encuentra presente en los partidos del América, estemos negando las graves consecuencias que esto presenta tanto para la sociedad como para el deporte.

5 Esta pregunta parte de que nuestro caso analizado en ningún momento nadie reclamo cuando se hacían insultos hacia las personas afrocolombianas. 
Por un lado, observamos y escuchamos que cuando algún jugador afrocolombiano resuelve bien una situación, o hace una jugada difícil de realizar, por lo general se dicen cosas como:

\author{
"Vamos mi negro". ${ }^{6}$ \\ "Bien! mi negro". \\ "Negrito: jel gol del ascenso es tuyo!"
}

No obstante, a diferencia de cuando se hacen comentarios de manera negativa, los comentarios que resaltan las habilidades de un jugador afro, no siempre hacen alusión a su color de piel sino a su apellido u otras referencias.

“Borja papasito!"” (después de una gran jugada). ${ }^{9}$

“Bejarano es mucho arquero". ${ }^{10}$

Asimismo, hay comentarios que hacen alusión al color de piel pero adicionalmente son acompañados de un insulto, de palabras despectivas, e incluso una de las expresiones más fuertes que se dieron durante los tres partidos, se dio cuando la hinchada imitó los sonidos de un gorila para hacer referencia a un jugador afrocolombiano del equipo rival:

$6 \quad$ Este comentario es constante en todos los partidos analizados en los cuadrangulares de ascenso, es importante resaltar que solo se asistió a los partidos de local, los otros datos recopilados fueron recopilados en la ciudad de Cali en distintos puntos de la ciudad (días 25 de octubre en el Estadio de Armenia; 01,06 y 27 de noviembre en el Pascual Guerrero; 13 de noviembre en el estadio de Cartagena; 20 de noviembre en el estadio de Popayán).

7 Comentario posterior a que Borja marcara el gol contra Cartagena el 6 de noviembre en el pascual.

8 Comentario dicho antes de que Martínez Borja pateara el penal que le daría el Ascenso al América, en la tribuna de oriental segundo piso, en el Pascual Guerrero, Cali, Valle.

9 Comentario posterior a que Borja hiciera una jugada de calidad contra Cartagena el 6 de noviembre en el pascual.

10 Minuto 92 aproximadamente, cuando América ganaba 2-1 y con ese resultado ascendía. Bejarano la sacó de la línea de meta. 27 de noviembre de 2016, Pascual Guerrero, tribuna de oriental segundo piso. 
Negro marica (al rival). ${ }^{11}$

Ug Ug Ug (como gorila) al jugador de Popayán. ${ }^{12}$

Por otro lado, de manera similar al estereotipo que menciona Bonilla (2011) respecto a las películas "juegan el papel de "negros mágicos", negros a los que se les da poder y cuyo trabajo en la trama de una película es ayudar a los blancos a navegar por sus vidas", así mismo en el futbol hay un estereotipo de que los jugadores afrocolombianos corren más rápido que los demás, tienen habilidad para driblar, regatear y jugar bonito, razón por la cual cuando hacen una jugada mal hecha, se alude a su color de piel para hacer énfasis en que debían hacerlo bien. No obstante, a pesar de que se espera que corran y driblen bien, en el imaginario construido en el futbol, los jugadores afrodescendientes no son buenos para "pensar" sino que sus virtudes radican en el físico, entonces el estereotipo que se tiene implica que sean malos a la hora de definir, o de hacer buenos pases y surgen comentarios como:

"Hasta que el América no arme un equipo de 90\% blancos no asciende". ${ }^{13}$

"Ese negro juega mucho". ${ }^{14}$

"América ya no tiene negrito y los que tiene son buenos". 15

"Negro tenía que ser". ${ }^{16}$

11 Comentario tras la salida del campo de Popayán a la gramilla del Estadio Pascual Guerrero el 01 de noviembre de 2016 .

12 Coreaban cuando el balón lo tenía un jugador afro de Popayán en la gramilla del Estadio Pascual Guerrero el 01 de noviembre de 2016.

13 Comentario escuchado en sitio de comida rápida de Cali, donde transmitieron el partido de Quindío vs América el 25 de octubre en Armenia.

14 Comentario posterior a una jugada de gran calidad de Lucumi -jugador del América-. en la tribuna de oriental segundo piso, en el Pascual Guerrero, Cali, Valle.

15 Momento previo a que iniciara el primer partido de cuadrangulares de ascenso en bienestar universitario en la Universidad Icesi.

16 Frase dicha luego que Bejarano -arquero del América- ocasionara un penal. Comentario escuchado en sitio de comida rápida de Cali, donde transmitieron el partido de Quindío vs. América el 25 de octubre en Armenia. 
Ahora bien, según Bonilla (2011) la gramática racial "normaliza los estándares de la supremacía blanca como estándares para todos tipo de transacciones y eventos sociales", lo cual en el Estadio se puede ver ejemplificado en aquellos insultos que van dirigidos hacia los jugadores del mismo equipo que se está apoyando. Puesto que si bien hemos hablado que las dinámicas propias del futbol y del estadio, generan en las personas una necesidad de agredir verbal al otro equipo, y bajo este marco surjan o se hagan explicitas expresiones racistas contra el equipo que es concebido como un enemigo, luego estas se hayan normalizado de manera tal que dejan de ser una estrategia para desconcentrar al rival, y se apliquen incluso contra los jugadores del mismo equipo.

\section{Conclusiones}

A manera de cierre, se puede afirmar que, el estadio es un espacio cuyas dinámicas de rivalidad promueven el uso de un lenguaje agresivo hacia el oponente, lo cual en este marco ha generado que se haga uso de expresiones de carácter racista como un insulto, que incluso haya sido normalizado a tal punto que dicha violencia verbal sea usada no solo con los jugadores del equipo rival, sino con el mismo equipo al cual se está apoyando. Ahora bien, esto parece ser un indicio del problema que se presenta cuando -ya sea por rivalidad o por otros factores- en determinado espacio se legitima el uso del racismo, puesto que se va reproduciendo como una conducta normal que tiende a salirse de dicho lugar, y a la vez, el poco cuidado que ha tenido las instituciones colombianas competentes para enfrentar esto.

\section{Referencias}

Bonilla-Silva, E (2012) The invisible weight of whiteness: the racial grammar of everyday life in contemporary America. Ethnic and Racial Studies 35(2): 173-194

González, J \& Miménez M. (2006). Fútbol y Racismo: un problema científico y social. International Journal of Sport Science, Vol II.

Lapchick, R.E. (2005) “Crime and Athletes. New Racial Stereotypes”, en D. Stanley Eitzen (ed.), Sport in Contemporary Society (pp. 187-198). New York, Worth Publishiers.

Mills, C. (1997). The Racial Contract. Editorial Cornell University Press. 
Muñoz, R. (2009). "Tarjeta Roja” Fútbol y racismo. La historia de vida del jugador Agustín Delgado y su probable victimización racial. Facultad Latinoamericana de Ciencias Sociales Sede Ecuador.

Raxen, Informe (2005). Racismo y Violencia Ultra en el Fútbol. Madrid, Movimiento contra la Intolerancia.

Redacción de El Tiempo (2015). Enredos entre Real Sincelejo y Dépor por una ficha en la Primera B. En El Tiempo, noviembre 4, 2015. Recuperado desde http://www. eltiempo.com/archivo/documento/CMS-16421351

Redacción de El País (2016). Atlético Fútbol Club, antes Dépor, ya tiene reconocimiento deportivo. En El País, Marzo 11, 2016. Recuperado desde http://www.elpais.com.co/ deportes/atletico-futbol-club-antes-depor-ya-tiene-reconocimiento-deportivo.html

Sage, G.H. (2001) "Racial Inequality and Sport", en D. Stanley Eitzen (ed.), Sport in Contemporary Society (pp. 275-284). New York, Worth Publishiers.

UNESCO (2016). ¿Color? ¿Qué color?, Informe sobre la lucha contra el racismo y la discriminación en el fútbol. Edición: Fundación Santillana 